\title{
ON ALGEBROID FUNCTIONS TAKING THE SAME VALUES AT THE SAME POINTS
}

\author{
By He YuZAN AND GaO SHI-AN
}

1. It is well known that if two meromorphic functions $w(z)$ and $\hat{w}(z)$ take five values at the same points, then $w(\boldsymbol{z}) \equiv \hat{w}(\boldsymbol{z})$, and if $w(\boldsymbol{z})$ and $\hat{w}(\boldsymbol{z})$ share only four values, usually $w(z) \not \equiv \hat{w}(z)$, but there exists some relations between $w(z)$ and $\hat{w}(z)$. Recently G. Gundersen [1] proved that if two meromorphic functions share three values, then the proportion of their characteristic functions is finite. On the $\nu$-valued algebroid functions G. Valiron [5] pointed out that if two $\nu$-valued algebroid functions $w(z)$ and $\hat{w}(z)$ take $4 \nu+1$ values at the same points with same multiple order, then $w(z) \equiv \hat{w}(z)$. In [3], we proved a uniqueness theorem which refined the result of Valiron. In present paper we first prove that if two $\nu$-valued algebroid functions $w(z)$ and $\hat{w}(z)$ share $4 \nu$ values, then there exists some relations between $w(z)$ and $\hat{w}(z)$, and we construct two different $\nu$-valued algebroid functions sharing $4 \nu$ values. Secondly we proved that if two $\nu$-valued algebroid functions share $2 \nu+\lambda$ values with $1 \leqq \lambda \leqq 2 \nu-1$, then the ratio of their characteristic functions is finite, and we give two $\nu$-valued algebroid functions sharing $2 \nu$ values, but the ratio of their characteristic functions is infinite. We also obtain some results concerning the multiplicity.

2. Let $w(z)$ be a $\nu$-valued algebroid function defined by the following irreducible equation

$$
\psi(z, w) \equiv A_{\nu}(z) w^{\nu}+A_{\nu-1}(z) w^{\nu-1}+\cdots+A_{0}(z)=0
$$

where $A_{\jmath}(z)(\jmath=0,1, \cdots, \nu)$ are entire functions in $\boldsymbol{C}$. Set

$$
\begin{aligned}
N\left(r, \frac{1}{w-a}\right)=\frac{1}{\nu} N\left(r, \frac{1}{\psi(z, a)}\right)=\frac{1}{\nu} \int_{0}^{r} \frac{n\left(t, \frac{1}{w-a}\right)-n\left(0, \frac{1}{w-a}\right)}{t} d t & \\
& \quad+\frac{1}{\nu} n\left(0, \frac{1}{w-a}\right) \log r, \quad a \in C
\end{aligned}
$$

and

$$
N(r, w)=\frac{1}{\nu} N\left(r, \frac{1}{A_{\nu}}\right)=\frac{1}{\nu} \int_{0}^{r} \frac{n(t, w)-n(0, w)}{t} d t+\frac{1}{\nu} n(0, w) \log r, \quad a=\infty
$$

Received November 18, 1985 
where $n\left(t, \frac{1}{w-a}\right)$ is the number of the zeroes of $w(z)-a$ in $|z|<t$ being counted the multiply. We denote by $J(z)=\left[A_{\nu}(z)\right]^{2(\nu-1)} \prod_{1 \leqq j<k \leqq \nu}\left[w_{j}(z)-w_{k}(z)\right]^{2}$ or

$$
J(z)=\left|\begin{array}{l}
1, A_{\nu-1}(z), \cdots, A_{0}(z), 0, \cdots, 0 \\
0, A_{\nu}(z), A_{1}(z), \cdots, A_{0}(z), 0, \cdots, 0 \\
\cdots \cdots \cdots \\
0, \cdots, 0, A_{\nu}(z), A_{\nu-1}(z), \cdots, A_{0}(z) \\
\nu,(\nu-1) A_{\nu-1}(z), \cdots, A_{1}(z), 0, \cdots, 0 \\
0, \nu A_{\nu}(z),(\nu-1) A_{\nu-1}(z), \cdots, A_{1}(z), 0, \cdots, 0 \\
\cdots \cdots \cdots \\
0, \cdots, 0, \nu A_{\nu}(z),(\nu-1) A_{\nu-1}(z), \cdots, A_{1}(z)
\end{array}\right| \nu
$$

the discriminant, and it is well known that each branch point of $w(z)$ is a zero of $J(z)$. Let $L$ denote a curve joining all zeroes of $J(z)$, then the determinations $w_{j}(z)$ of $w(z)(j=1,2, \cdots, \nu)$ are simple-valued functions in $C \backslash L$. We set

$$
m\left(r, \frac{1}{w-a}\right)=\frac{1}{\nu} \sum_{j=1}^{\nu} m\left(r, \frac{1}{w_{j}-a}\right)=\frac{1}{\nu} \sum_{j=1}^{\nu} \frac{1}{2 \pi} \int_{0}^{2 \pi} \log ^{+} \frac{1}{\left|w_{j}\left(r e^{2 \varphi}\right)-a\right|} d \varphi,
$$

and

$$
a \in C
$$

$$
m(r, w)=\frac{1}{\nu} \sum_{\jmath=1}^{\nu} m^{\prime}\left(r, w_{\jmath}\right)=\frac{1}{\nu} \sum_{\jmath=1}^{\nu} \frac{1}{2 \pi} \int_{0}^{2 \pi} \log ^{+}\left|w_{\jmath}\left(r e^{2 \varphi}\right)\right| d \varphi, \quad a=\infty
$$

and we call

$$
T(r, w)=m(r, w)+N(r, w)
$$

the characteristic function of $w(z)$. We have (cf. [5] or [6])

ThEOREM A. (The first fundamental theorem). If $a$ is any complex number, then

$$
m\left(r, \frac{1}{w-a^{*}}\right)+N\left(r, \frac{1}{w-a}\right)=T(r, w)+\frac{1}{\nu} \log \left|\frac{\psi(0, a)}{A_{\nu}(0)}\right|+\epsilon(r, a),
$$

where

$$
|\epsilon(r, a)| \leqq \log ^{+}|a|+\log 2
$$

Theorem B. (The second fundamental theorem). Let $w(z)$ be a v-valued algebroid function and $a_{j} \in \hat{\boldsymbol{C}}(j=1,2, \cdots, p)$ be $p$ different number, then

$$
(p-2 v) T(r, w)<\sum_{j=1}^{p} N\left(r, \frac{1}{w-a,}\right)-N_{1}(r, w)+S(r, w)
$$

where $N_{1}(r, w)$ is the counting function for all multiple value-ponts of $w(z)$, but a $\tau$-fold value-point is counted only $\tau-1$ times and $S(r, w)$ is the remainder term.

We denote by $\bar{n}\left(t, \frac{1}{w-a}\right)$ the number of distinct roots of $w(z)=a$ in $|z|<t$ 
and define

$$
\bar{N}\left(r, \frac{1}{w-a}\right)=\frac{1}{\nu} \int_{0}^{r} \frac{\bar{n}\left(t, \frac{1}{w-a}\right)-\bar{n}\left(0, \frac{1}{w-a}\right)}{t} d t+\frac{1}{\nu} \bar{n}\left(0, \frac{1}{w-a}\right) \log r
$$

then the second fundamental theorem can be written as the form.

THEOREM B'. Suppose $w(z)$ and $a_{\jmath} \in C$ are the same as theorem $B$, then

$$
(p-2 \nu) T(r, w)<\sum_{j=1}^{\phi} \bar{N}\left(r, \frac{1}{w-a,}\right)+O\{\log (r T(r, w))\}
$$

outside a certain exceptional set of finite linear measure.

Let $\vec{E}(a, w)$ denote the set of distinct roots of $w(z)=a$, then we have and

THEOREM 1. Let $w(z)$ and $\hat{w}(z)$ be two algebroid functions definied by (1)

$$
\Phi(z, \hat{w}) \equiv B_{\nu}(z) \hat{w}^{\nu}+B_{\nu-1}(z) \hat{w}^{\nu-1}+\cdots+B_{0}(z)=0
$$

respectively. If $\bar{E}\left(a_{\jmath}, w\right)=\bar{E}\left(a_{\jmath}, \hat{w}\right)$ for $a_{j} \in \hat{\boldsymbol{C}},(j=1,2, \cdots, 4 \nu)$, then it must be

$$
\begin{gathered}
\lim _{\substack{r \rightarrow \infty \\
r \in E}} \frac{T(r, w)}{T(r, \hat{w})}=1, \\
\lim _{\substack{r \rightarrow \infty \\
r \in E}} \sum_{j=1}^{4 \nu} \frac{\bar{N}\left(r, \frac{1}{w-a_{\jmath}}\right)}{T(r, w)}=\lim _{\substack{r \rightarrow \infty \\
r \in E}} \sum_{j=1}^{4 \nu} \frac{\bar{N}\left(r, \frac{1}{\hat{w}-a_{\jmath}}\right)}{T(r, \hat{w})}=2 \nu,
\end{gathered}
$$

(iii) for any $a \neq a$, then

$$
\lim _{\substack{r \rightarrow \infty \\ r \in E}} \frac{\bar{N}\left(r, \frac{1}{w-a}\right)}{T(r, w)}=\lim _{\substack{r \rightarrow \infty \\ r \in E}} \frac{\bar{N}\left(r, \frac{1}{\hat{w}-a}\right)}{T(r, \hat{w})}=1
$$

where $E$ is a set with finite linear measure.

Proof. (i) Suppose $w(z) \not \equiv \hat{w}(z)$ and $\bar{n}_{0}(t, a)$ denotes the number of the common roots of $w(z)=a$ and $\hat{w}(z)=a$ containing in $|z|<t$ and each common root is counted only once. We define

$$
\bar{N}_{0}(r, a)=\frac{1}{2} \int_{0}^{r} \frac{\bar{n}_{0}(t, a)-\bar{n}_{0}(0, a)}{t} d t+\frac{1}{\nu} \bar{n}_{0}(0, a) \log r .
$$

It is easy to know that

$$
\sum \bar{n}_{0}\left(r, a_{j}\right) \leqq n\left(r, \frac{1}{R(\phi, \Phi)}\right)
$$

where $R(\psi, \Phi)$ is the resultant of (1) and $(1)^{\prime}$, i. e. 


$$
\left.\begin{array}{rl}
R(\psi, \Phi)= & {\left[A_{\nu}(z) B_{\nu}(z)\right]^{\nu} \prod_{\substack{1 \leq j \leq \nu \\
1 \leq k \leq \nu}}\left[w_{j}(z)-\hat{w}_{k}(z)\right]} \\
& =\left|\begin{array}{l}
A_{\nu}(z), A_{\nu-1}(z), \cdots, A_{0}(z), 0, \cdots, 0 \\
0, A_{\nu}(z), A_{\nu-1}(z), \cdots, A_{0}(z), 0, \cdots, 0 \\
\cdots \cdots \cdots \\
0, \cdots, 0, A_{\nu}(z), A_{\nu-1}(z), \cdots, A_{0}(z) \\
B_{\nu}(z), B_{\nu-1}(z), \cdots, B_{0}(z), 0, \cdots, 0 \\
0, B_{\nu}(z), B_{\nu-1}(z), \cdots, B_{0}(z), 0, \cdots, 0 \\
\cdots \cdots \cdots \\
0, \cdots, 0, B_{\nu}(z), B_{\nu-1}(z), \cdots, B_{0}(z)
\end{array}\right|
\end{array}\right\} \nu
$$

by using the Jensen's formula we get

hence

$$
\begin{aligned}
N\left(r, \frac{1}{R(\phi, \Phi)}\right)= & \frac{1}{2 \pi} \int_{0}^{2 \pi} \log |R(\psi, \Phi)| d \varphi+\log \left|\frac{1}{R(\phi, \Phi)}\right|_{z=0} \\
= & \frac{\nu}{2 \pi} \int_{0}^{2 \pi} \log \left|A_{\nu}\left(r e^{\imath \varphi}\right)\right| d \varphi+\frac{\nu}{2 \pi} \int_{0}^{2 \pi} \log \left|B_{\nu}\left(r e^{\imath \varphi}\right)\right| d \varphi \\
& +\frac{1}{2 \pi} \int_{0}^{2 \pi} \log \left|\prod_{\substack{1 \leq s \leq \nu \\
1 \leqq k \leqq \nu}}\left[w_{j}\left(r e^{\imath \varphi}\right)-\hat{w}_{k}\left(r e^{\imath \varphi}\right)\right]\right| d \varphi+\log \left|\frac{1}{R(\phi, \Phi)}\right|_{z=0} \\
\leqq & \nu\left(r, \frac{1}{A_{\nu}}\right)+\nu N\left(r, \frac{1}{B_{\nu}}\right)+\nu \sum_{j=1}^{\nu} m\left(r, w_{j}\right)+\nu \sum_{k=1}^{\nu} m\left(r, \hat{w}_{k}\right)+O(1) \\
= & \nu^{2}[T(r, w)+T(r, \hat{w})]+O(1)
\end{aligned}
$$

$$
\sum_{j=1}^{4 \nu} \bar{N}_{0}\left(r, a_{j}\right) \leqq \nu[T(r, w)+T(r, \hat{w})]+O(1)
$$

applying Theorem $\mathrm{B}^{\prime}$ to $w(z),\left\{a_{j}\right\}$ and $\hat{w}(z),\left\{a_{j}\right\}$, we have

$$
2 \nu T(r, w)<\sum_{j=1}^{4 \nu} \bar{N}\left(r, \frac{1}{w-a_{\jmath}}\right)+O\{T(r, w)\}
$$

and

$$
2 \nu T(r, \hat{w})<\sum_{j=1}^{4 \nu} \bar{N}\left(r, \frac{1}{\hat{w}-a_{\jmath}}\right)+O\{T(r, \hat{w})\}
$$

outside of a possible exceptional set $E$ with a finite linear measure. Since $\bar{N}\left(r, \frac{1}{w-a_{j}}\right)=\bar{N}_{0}\left(r, a_{j}\right)$, noting (8) we get

$$
\begin{aligned}
2 \nu T(r, w) & <\sum_{j=1}^{4 \nu} \bar{N}_{0}\left(r, a_{j}\right)+O\{T(r, w)\} \\
& \leqq \nu[T(r, w)+T(r, \hat{w})]+O\{T(r, w)\}
\end{aligned}
$$

thus

$$
T(r, w)<T(r, \hat{w})+O\{T(r, w)\}
$$

by a similar argument we have 


$$
T(r, \hat{w})<T(r, w)+O\{T(r, \hat{w})\}
$$

From (10) and (11), it follows (5).

(ii) Combining (4)' with (9) and (11) we get

$$
\begin{aligned}
(2 \nu+O(1)) T(r, w) & \leqq \sum_{j=1}^{4 \nu} \bar{N}\left(r, \frac{1}{w-a_{\jmath}}\right)=\sum_{j=1}^{4 \nu} \bar{N}_{0}\left(r, a_{j}\right) \\
& \leqq(2 \nu+O(1)) T(r, w)
\end{aligned}
$$

it shows that the first equality of (6) holds. Similarly we have the second one of (6).

(iii) Applying the theorem $\mathrm{B}^{\prime}$ to $w(z), a_{\jmath}(\jmath=1,2, \cdots, 4 \nu)$ and $a$, and noting (8) and (11) we obtain

$$
\begin{aligned}
(2 \nu+1) T(r, w) & <\sum_{\jmath=1}^{4 \nu} \bar{N}\left(r, \frac{1}{w-a_{\jmath}}\right)+\bar{N}\left(r, \frac{1}{w-a}\right)+S(r, w) \\
& =\sum_{\jmath=1}^{4 \nu} \bar{N}_{0}\left(r, a_{\jmath}\right)+\bar{N}\left(r, \frac{1}{w-a}\right)+O\{T(r, w)\} \\
& =(2 \nu+O(1)) T(r, w)+\bar{N}\left(r, \frac{1}{w-a}\right)
\end{aligned}
$$

therefore

$$
T(r, w) \leqq \bar{N}\left(r,-\frac{1}{w-a}\right)+O\{T(r, w)\} \leqq(1+O(1)) T(r, w)
$$

Similarly

$$
T(r, \hat{w}) \leqq \bar{N}\left(r, \frac{1}{\hat{w}-a}\right)+O\{T(r, w)\} \leqq(1+O(1)) T(r, \hat{w})
$$

it follows (7).

Now we give two different $\nu$-valued algebroid functions which take $4 \nu$ values at the same points and satisfy the conclusions given by Theorem 1 .

Let $w(z)$ and $\hat{w}(z)$ be two $\nu$-valued algebroid functions defined by

and

$$
\psi(z, w) \equiv\left(a+b c^{z}\right) w^{\nu}-\left(c+d e^{z}\right)=0
$$

$$
\Phi(z, \hat{w}) \equiv\left(a+b c^{-z}\right) \hat{w}^{\nu}-\left(c+d e^{-z}\right)=0
$$

respectively, where $a, b, c$ and $d$ are different non-zero complex numbers with $a d-b c \neq 0$ and $\frac{c+d}{a+b} \neq 0, \infty$.

It is obvious that $w(\boldsymbol{z}) \not \equiv \hat{w}(\boldsymbol{z})$. Suppose $a_{\jmath}=\left|\frac{c}{a}\right|^{1 / \nu} e^{i(\alpha / \nu+2 \pi j / \nu)}(\jmath=1,2, \cdots, \nu)$ with $\quad \alpha=\arg \frac{c}{a}, \quad b_{k}=\left|\frac{d}{b}\right|^{1 / \nu} e^{i(\beta / \nu+2 k \pi / \nu)} \quad(k=1,2, \cdots, \nu)$ with $\quad \beta=\arg \frac{d}{b}, \quad c_{l}=$ $\left|\frac{c+d}{a+b}\right|^{1 / \nu} e^{i(\gamma / \nu+2 \pi l / \nu)}(l=1,2, \cdots, \nu)$ with $\gamma=\arg \frac{c+d}{a+b}, \quad d_{m}=\left|\frac{c-d}{a-b}\right|^{1 / \nu} e^{i(\delta / \nu+2 \pi m / \nu)}$ $(m=1,2, \cdots, \nu)$ with $\delta=\arg \frac{c-d}{a-b}$. IWe can show that $\bar{E}\left(a_{\jmath}, w\right)=\bar{E}\left(a_{\jmath}, \hat{w}\right), \bar{E}\left(b_{k}, w\right)$ $=\bar{E}\left(b_{k}, \hat{w}\right), \quad \bar{E}\left(c_{l}, w\right)=\bar{E}\left(c_{l}, \hat{w}\right), \quad \bar{E}\left(d_{m}, w\right)=\bar{E}\left(d_{m}, \hat{w}\right), \quad(j, k, l, m=1,2, \cdots, \nu)$. 
Noting the roots of $w(z)=a$ (or $\hat{w}(z)=a$ ) are the zeroes of $\phi(z, a)=0$ (or $\Phi(z, a)$ $=0$ ), we have

$$
\psi\left(z, a_{j}\right)=\frac{b c-a d}{a} e^{z} \neq 0, \quad(j=1,2, \cdots, \nu)
$$

thus $a,(j=1,2, \cdots, \nu)$ are the Picard exceptional values of $w(z)$ i.e. $\bar{E}\left(a_{\jmath}, w\right)$ $=\varnothing(j=1,2, \cdots, \nu)$. Similarly

$$
\Phi\left(z, a_{j}\right)=\frac{b c-a d}{a} e^{-z} \neq 0
$$

$a_{\jmath}(j=1,2, \cdots, \nu)$ are also the Picard exceptional values of $\hat{w}(z)$ i. e. $\bar{E}\left(a_{\jmath}, \hat{w}\right)$ $=\varnothing(j=1,2, \cdots, \nu)$. For $b_{k}(k=1,2, \cdots, \nu)$, because

$$
\phi\left(z, b_{k}\right)=a-c=\Phi\left(z, b_{k}\right)
$$

we have $b_{k}(k=1,2, \cdots, \nu)$ are also the Picard exceptional values of $w(z)$ and $\hat{w}(z)$, and therefore $\bar{E}\left(b_{k}, w\right)=\bar{E}\left(b_{k}, \hat{w}\right)=\varnothing(k=1,2, \cdots, \nu)$. For $c_{l}(l=1,2, \cdots, \nu)$, since

$$
\phi\left(z, c_{l}\right)=\frac{a d-b c}{a+b}\left(1-e^{z}\right),
$$

we have $\bar{E}\left(c_{l}, w\right)=\{2 \pi n i, n \in \boldsymbol{Z}\}$, on the other hand, since

$$
\Phi\left(z, c_{l}\right)=\frac{a d-b c}{a+b}\left(1-e^{-z}\right)
$$

we have $\bar{E}\left(c_{l}, \hat{w}\right)=\{2 \pi n i, n \in \boldsymbol{Z}\}$, it shows that $\bar{E}\left(c_{l}, w\right)=\bar{E}\left(c_{l}, \hat{w}\right)(l=1,2, \cdots, \nu)$. Finally for $d_{m}$, since

$$
\phi\left(z, d_{m}\right)=-\frac{a d-b c}{a-b}\left(1+e^{z}\right)
$$

and

$$
\Phi\left(z, d_{m}\right)=-\frac{a d-b c}{a-b}\left(1+e^{-z}\right)
$$

$(m=1,2, \cdots, \nu)$, we get $\bar{E}\left(d_{m}, w\right)=\bar{E}\left(d_{m}, \hat{w}\right)=\{(2 n+1) \pi i, n \in \boldsymbol{Z}\}$. It shows that $w(z)$ and $\hat{w}(z)$ take $4 \nu$ values at the same points, but $w(z) \not \equiv \hat{w}(z)$.

We can point out that $w(z)$ and $\hat{w}(z)$ satisfy the conclusions of theorem 1 . In fact, it is easy to show that

$$
T(r, w)=\frac{r}{\nu \pi}+O(1) \text { and } T(r, \hat{w})=\frac{r}{\nu \pi}+O(1)
$$

thus

$$
\lim _{r \rightarrow \infty} \frac{T(r, w)}{T(r, \hat{w})}=1
$$

since $\bar{N}_{0}\left(r, a_{j}\right)=\bar{N}_{0}\left(r, b_{k}\right)=0,(j, k=1,2, \cdots, \nu), \bar{N}_{0}\left(r, c_{l}\right)=\frac{r}{\nu \pi}+O(1)$ and $\bar{N}_{0}\left(r, d_{m}\right)$ 
$=\frac{r}{\nu \pi}+O(1),(l, m=1,2, \cdots, \nu)$, we get

$$
\begin{aligned}
& \lim _{r \rightarrow \infty} \sum_{j=1}^{\nu} \frac{\mid \bar{N}_{0}\left(r, a_{j}\right)+\bar{N}_{0}\left(r, b_{j}\right)+\bar{N}_{0}\left(r, c_{j}\right)+\bar{N}_{0}\left(r, d_{j}\right)}{T(r, w)} \\
= & \lim _{r \rightarrow \infty} \sum_{j=1}^{\nu} \frac{\bar{N}_{0}\left(r, a_{j}\right)+\bar{N}_{0}\left(r, b_{j}\right)+\bar{N}_{0}\left(r, c_{j}\right)+\bar{N}_{0}\left(r, d_{j}\right)}{T(r, \hat{w})}=2 \nu .
\end{aligned}
$$

Finally if $s \neq a_{\jmath}, b_{k}, c_{l}$ and $d_{m}$, then $\frac{c-a s^{\nu}}{b s^{\nu}-d} \neq 0, \infty$, since

$$
\phi(z, s)=\left(a+b e^{z}\right) s^{\nu}-\left(c+d e^{z}\right)=0 \quad \text { and } \quad \Phi(z, s)=\left(a+b e^{-z}\right) s^{\nu}-\left(c+d e^{-z}\right)=0
$$

we get $\bar{E}(s, w)=\left\{\log \frac{c-a s^{\nu}}{b s^{\nu}-d}+2 n \pi i, n \in \boldsymbol{Z}\right\}$ and $\bar{E}(s, \hat{w})=\left\{\log \frac{b s^{\nu}-d}{c-a s^{\nu}}+2 n \pi i\right.$, $n \in \boldsymbol{Z}\}$ therefore $\bar{N}\left(r, \frac{1}{w-s}\right)=\frac{r}{\nu \pi}+O(1)$ and $\bar{N}\left(r, \frac{1}{\hat{w}-s}\right)=\frac{r}{\nu \pi}+O(1)$, it follows that

$$
\lim _{r \rightarrow \infty} \frac{\bar{N}\left(r, \frac{1}{w-s}\right)}{T(r, w)}=\lim _{r \rightarrow \infty} \frac{\bar{N}\left(r, \frac{1}{\hat{w}-s}\right)}{T(r, \hat{w})}=1
$$

it shows that $w(z)$ and $\hat{w}(z)$ satisfy all conclusions in theorem 1.

THEOREM 2. Let $w(z)$ and $\hat{w}(z)$ be two v-valued algebroid functions, if $\bar{E}\left(a_{\jmath}, w\right)=\bar{E}\left(a_{\jmath}, \hat{w}\right)$ for $a_{j} \in \hat{\boldsymbol{C}}(j=1,2, \cdots, 2 \nu+\lambda)$ with $1 \leqq \lambda \leqq 2 \nu-1$, then there exists a finite non-zero number $K=K(\nu, \lambda)$ such that

$$
\frac{1}{K} \leqq \lim _{\substack{r \rightarrow \infty \\ r \in E}} \frac{T(r, w)}{T(r, \hat{w})} \leqq K
$$

where $E$ denotes a set with a finite linear mearsure.

Proof. Applying theorem $\mathrm{B}^{\prime}$ to $w(z),\left\{a_{j}\right\},(j=1,2, \cdots, 2 \nu+\lambda)$, we get

$$
\lambda T(r, w)<\sum_{j=1}^{2 \nu+\lambda} \bar{N}\left(r, \frac{1}{w-a,}\right)+S(r, w)
$$

noting $\bar{N}\left(r, \frac{1}{w-a_{\jmath}}\right)=\bar{N}\left(r, \frac{1}{\hat{w}-a_{\jmath}}\right) \leqq T(r, \hat{w})+O(1)$, we have

$$
\begin{aligned}
\lambda T(r, w) & <\sum_{j=1}^{2 \nu+\lambda} \bar{N}\left(r, \frac{1}{\hat{w}-a \jmath}\right)+O\{T(r, w)\} \\
& \leqq(2 \nu+\lambda) T(r, \hat{w})+O\{T(r, w)\}
\end{aligned}
$$

then for any $\epsilon>0$, there exists $r_{0}$ such that if $r \geqq r_{0}$ and $r \bar{E} E$, we have

$$
\left(\frac{\lambda}{2 \nu+\lambda}-\epsilon\right) T(r, w)<T(r, \hat{w}) \text {. }
$$


Similarly we get

$$
\left(\frac{\lambda}{2 \gamma+\lambda}-\epsilon\right) T(r, \hat{w})<T(r, w)
$$

where $E$ is a set of finite linear measure. Thus

$$
\left(\frac{\lambda}{2 \nu+\lambda}-\epsilon\right) \leqq \lim _{\substack{r \rightarrow \infty \\ r \in E}} \frac{T(r, w)}{T(r, \hat{w})} \leqq\left(\frac{\lambda}{2 \nu+\gamma}-\epsilon\right)^{-1}
$$

because of the arbitrariness of $\epsilon$, it follows (12).

The following example shows that if two $\nu$-valued algebroid functions take only $2 \nu$ values at the same points, then the ratio of their characteristic functions may be infinite. Let $w(z)$ and $w(z)$ be two algebroid functions defined by

and

$$
\phi(z, w) \equiv\left(1+e^{z}\right) w^{\nu}-\left(a+b e^{z}\right)=0
$$

$$
\Phi(z, \hat{w}) \equiv\left(1+e^{e^{z}}\right) \hat{w}^{\nu}-\left(a+b^{e^{z}}\right)=0
$$

respectivelly, where $a$ and $b$ are non-zero and distinct complex numbers. Set $a=|a| e^{\imath \alpha}$ and $a_{j}=|a|^{1 / \nu} e^{i(\alpha / \nu+2 \pi j / \nu)}, \quad(j=1,2, \cdots, \nu), \quad b=|b| e^{i \beta}$ and $b_{k}=$ $|b|^{1 / \nu} e^{i(\beta / \nu+2 \pi k / \nu)},(k=1,2, \cdots, \nu)$. Since $\phi\left(z, a_{j}\right)=(a-b) e^{z} \neq 0, a,(j=1,2, \cdots, \nu)$ are the Picard exceptional values of $w(z)$, i. e. $\bar{E}\left(a_{\jmath}, w\right)=\varnothing$. On the other hand, $\Phi\left(z, a_{j}\right)=(a-b) e^{e^{z}} \neq 0$, it means $\bar{E}\left(a_{\jmath}, \hat{w}\right)=\varnothing$, hence $\bar{E}\left(a_{\jmath}, w\right)=\bar{E}\left(a_{\jmath}, \hat{w}\right), \quad(j=1$, $2, \cdots, \nu)$. Because $\phi\left(z, b_{k}\right)=\Phi\left(z, b_{k}\right)=b-a, k=1,2, \cdots, \nu$, thus again $\bar{E}\left(b_{k}, w\right)$ $=\bar{E}\left(b_{k}, \hat{w}\right), k=1,2, \cdots, \nu$. In other words, $w(z)$ and $\hat{w}(z)$ share $2 \nu$ values. But it is easy to show that $T(r, w)=\frac{r}{\nu \pi}+O(1)$ and $T(r, \hat{w})=\frac{e^{r}}{\nu \sqrt{ } 2 \pi^{3} r}(1+O(1))$ (cf. Hayman [2]), it follows that $\frac{T(r, \hat{w})}{T(r, w)} \rightarrow \infty$, as $r \rightarrow \infty$.

3. Let $\gamma(\geqq 1)$ be an integer and $\bar{E}^{r}(a, w)$ be the set of the distinct zeroes of $w(z)-a$ which multiple order $\leqq \gamma$. We denote by $\bar{N}^{r}\left(r, \frac{1}{w-a}\right)$ the counting function of the corresponding $a$-points of $w(z)$. We have

THEOREM 3. Let $w(z)$ and $\hat{w}(z)$ be two v-valued algebroid functions and $\gamma$ $(\geqq 1)$ be an integer which divides exactly $2 \nu$. If for $a_{j} \in \hat{\boldsymbol{C}}, \bar{E}^{\gamma}\left(a_{\jmath}, w\right)=\bar{E}^{\gamma}\left(a_{\jmath}, \widehat{w}\right)$, $j=1,2, \cdots, p_{r}, p_{r}=4 \nu+\frac{2 \nu}{\gamma}$, then

$$
\begin{gathered}
\lim _{\substack{r \rightarrow \infty \\
r \in E}} \frac{T(r, w)}{T(r, \hat{w})}=1 \\
\lim _{\substack{r \rightarrow \infty \\
r \in E}} \sum_{j=1}^{p_{\gamma}} \frac{\bar{N}^{r}\left(r, \frac{1}{w-a_{\jmath}}\right)}{T(r, w)} \lim _{\substack{r \rightarrow \infty \\
r \in E}} \sum_{j=1}^{p_{\gamma}} \frac{\bar{N}^{r}\left(r, \frac{1}{\hat{w}-a_{\jmath}}\right)}{T(r, \hat{w})}=2 \nu
\end{gathered}
$$


and (iii) for $a \neq a_{\jmath}$, we have

$$
\lim _{\substack{r \rightarrow \infty \\ r \in E}} \frac{\bar{N}^{r}\left(r, \frac{1}{w-a}\right)}{T(r, w)} \lim _{\substack{r \rightarrow \infty \\ r \in E}} \frac{\bar{N}^{r}\left(r, \frac{1}{\hat{w}-a}\right)}{T(r, \hat{w})}=1
$$

where $E$ is a set with finite linear measure.

Especially, if $\gamma=1,2, \cdots, \nu$, then $p_{r}=6 \nu, 5 \nu, 4 \nu+2$, respectively.

Proof. Set

$$
\bar{N}^{(r}\left(r, \frac{1}{w-a}\right)=\bar{N}\left(r, \frac{1}{w-a}\right)-\bar{N}^{r}\left(r, \frac{1}{w-a}\right)
$$

it is easy to know that $\bar{N}^{\prime r}\left(r, \frac{1}{w-a}\right) \leqq \frac{1}{r+1} N^{r r}\left(r, \frac{1}{w-a}\right)$ where $N^{<r}\left(r, \frac{1}{w-a}\right)$ is the counting function of the zeroes of $w(z)-a$ which multiple order $>\gamma$ and being counted multiply. Since

$$
\begin{aligned}
\bar{N}\left(r, \frac{1}{w-a}\right) & \leqq \bar{N}^{r}\left(r, \frac{1}{w-a}\right)+\frac{1}{\gamma+1} N^{(r}\left(r, \frac{1}{w-a}\right) \\
& \leqq \frac{\gamma}{\gamma+1} \bar{N}^{r}\left(r, \frac{1}{w-a}\right)+\frac{1}{\gamma+1} N\left(r, \frac{1}{w-a}\right) \\
& \leqq \frac{\gamma}{\gamma+1} \bar{N}^{r}\left(r, \frac{1}{w-a}\right)+\frac{1}{\gamma+1} T(r, w)+O(1)
\end{aligned}
$$

(4) can be written as the following form

$$
\begin{aligned}
(p-2 \nu) T(r, w) & <\sum_{\jmath=1}^{p} \bar{N}\left(r, \frac{1}{w-a_{\jmath}}\right)+S(r, w) \\
& \leqq \frac{\gamma}{\gamma+1} \sum_{\jmath=1}^{p} \bar{N}^{r}\left(r, \frac{1}{w-a_{\jmath}}\right)+\frac{p}{\gamma+1} T(r, w)+S(r, w)
\end{aligned}
$$

thus (4) becomes

$$
(p \gamma-2 \nu(\gamma+1)) T(r, w)<\gamma \sum_{j=1}^{p} \bar{N}^{r}\left(r, \frac{1}{w-a_{\jmath}}\right)+S_{\gamma}(r, w),
$$

By using (13) to $w(z),\left\{a_{j}\right\}$ and $\hat{w}(z),\left\{a_{j}\right\}, j=1,2, \cdots, p_{\gamma}$ we get (13) and

$$
\left(p_{\gamma} \gamma-2 \nu(\gamma+1)\right) T(r, \hat{w})<\gamma \sum_{j=1}^{p_{\gamma}} \bar{N}^{r}\left(r, \frac{1}{\hat{w}-a_{\jmath}}\right)+S_{\gamma}(r, \hat{w}) .
$$

By a argument similar to the proof of theorem 1 , we can prove theorem 3 .

Similarly we have the following

THEOREM 4. Let $w(z)$ and $\hat{w}(z)$ be two $\nu$-valued algebroid functions and $\gamma$ $(\geqq 1)$ an integer which divides exactly $2 \nu$, if $\bar{E}^{r}\left(a_{\jmath}, w\right)=\bar{E}^{r}\left(a_{\jmath}, \hat{w}\right)$ for $a_{\jmath} \in \hat{\boldsymbol{C}}$, $j=1,2, \cdots, p_{\gamma \lambda}, p_{\gamma \lambda}=2 \nu+\lambda+\frac{2 \nu}{\gamma}$ with $1 \leqq \lambda \leqq 2 \nu-1$, then 


$$
\frac{\lambda \gamma}{2 \nu(\gamma+1)+\lambda \gamma} \leqq \lim _{\substack{r \rightarrow \infty \\ r \in E}} \frac{T(r, w)}{T(r, \hat{w})} \leqq \frac{2 \nu(\gamma+1)+\lambda \gamma}{\lambda \gamma} .
$$

Especially, if $\gamma=1,2$ and $\nu$, then $p_{\gamma \lambda}=4 \nu+\lambda, 3 \nu+\lambda$ and $2(\nu+1)+\lambda$.

The authors wish to thank to Professor K. Niino for his valuable advice and careful examination.

\section{REFERENCES}

[1] G.G. Gundersen, Meromorphic functions that share three or four values, J. London Math. Soc. 20 (1979), 457-466.

[2] W.K. Hayman, Meromorphic functions, Oxford, 1964.

[3] He Yuzan, Sur un Problème d'unicité relatif aux fonctions algébroïdes, Sci. Sinica, 14 (1965), 174-180.

[4] He Yuzan, On multiple values of algebroid functions, Acta Math. Sinica 22 (1979), 733-742.

[5] E. UllRich, Über den Einfluss der verzwiegtheit einer Algebroide auf ihre Weitverteilung, J. Reine Ang. Math. 169 (1931), 198-220.

[6] G. Valiron, Sur la dérivée der fonctions algébroïdes, Bull. Soc. Math. Fr. 59 (1929), 17-39.

[7] G. Valikon, Sur quelques propriétés des fonctions algébroïdes, C.R. Acad. Sci. Paris 189 (1929), 824-826.

Institute of Mathematics Academia Sinica Beijing CHINA
Department of Mathematics South China Normal University ChINA 